\title{
Effect of population screening for type 2 diabetes on mortality: long-term follow-up of the Ely cohort
}

\author{
R. K. Simmons • M. Rahman • R. W. Jakes • \\ M. F. Yuyun • A. R. Niggebrugge • S. H. Hennings • \\ D. R. R. Williams • N. J. Wareham • S. J. Griffin
}

Received: 7 July 2010 /Accepted: 27 September 2010 /Published online: 27 October 2010

(C) Springer-Verlag 2010

\begin{abstract}
Aims/hypothesis The aim of this study was to assess the impact of invitation to screening for type 2 diabetes and related cardiovascular risk factors on population mortality. Methods This was a parallel-group population-based cohort study including all men and women aged 40-65 years, free of known diabetes, registered with a single practice in Ely, UK $(n=4,936)$. In 1990-1992, approximately one-third ( $n=$ $1,705)$ were randomly selected to receive an invitation to screening for diabetes (with an OGTT) and related cardiovascular risk factors. In the remaining two-thirds of
\end{abstract}

R. K. Simmons and M. Rahman contributed equally to this study.

R. K. Simmons • R. W. Jakes • S. H. Hennings • N. J. Wareham • S. J. Griffin $(\square)$

MRC Epidemiology Unit, Institute of Metabolic Science,

Box 285, Addenbrooke's Hospital, Hills Road,

Cambridge CB2 0QQ, UK

e-mail: simon.griffin@mrc-epid.cam.ac.uk

M. Rahman

General Practice \& Primary Care Research Unit,

University of Cambridge,

Cambridge, UK

M. F. Yuyun

Department of Public Health \& Primary Care,

Institute of Public Health, University of Cambridge,

Cambridge, UK

\section{A. R. Niggebrugge}

Eastern Region Public Health Observatory,

Institute of Public Health,

Cambridge, UK

D. R. R. Williams

Centre for Health Information, Research and Evaluation,

School of Medicine, University of Swansea,

Swansea, UK the population, 1,705 individuals were randomly selected for invitation to screening in 2000-2003 and 1,526 were not invited at any point during the follow-up period. All individuals were flagged for mortality until January 2008. Results There were 345 deaths between 1990 and 1999 (median 10 years follow-up). Compared with those not invited, individuals who were invited to the 1990-1992 screening round had a non-significant $21 \%$ lower all-cause mortality (HR 0.79 [95\% CI 0.63-1.00], $p=0.05$ ) after adjustment for age, sex and deprivation. There were 291 deaths between 2000 and 2008 (median 8 years follow-up), with no significant difference in mortality between invited and non-invited participants in 2000-2003. Compared with the non-invited group, participants who attended for screening at any time point had a significantly lower mortality and those who did not attend had a significantly higher mortality.

Conclusions/interpretation Invitation to screening was associated with a non-significant reduction in mortality in the Ely cohort between 1990 and 1999, but this was not replicated in the period 2000-2008. This study contributes to the evidence concerning the potential benefits of population screening for diabetes and related cardiovascular risk factors.

Keywords Cardiovascular disease · Diabetes · Ely Mortality $\cdot$ Population $\cdot$ Screening

$\begin{array}{ll}\text { Abbreviations } \\ \text { CVD } & \text { Cardiovascular disease } \\ \text { GP } & \text { General practitioner } \\ \text { ICD-10 } & \begin{array}{l}\text { International Classification of Diseases } \\ \left(10^{\text {th }} \text { edition }\right)\end{array} \\ \text { ONS } & \text { Office of National Statistics }\end{array}$




\section{Introduction}

Type 2 diabetes meets many of the criteria for suitability for screening. It is increasingly common and creates a substantial burden of suffering and health service use [1]. The high proportion of undiagnosed cases [2], the long latent period of the disease [3], and the high proportion of individuals with complications at diagnosis [4] are strong arguments for diabetes screening. As such, population screening has been recommended by some national organisations and the NHS plans to include tests for diabetes in its health checks programme for all individuals over 40 years of age in the UK [5]. However, significant uncertainties remain [6].

Some of the uncertainties regarding population screening for diabetes have recently been reduced. There is now evidence from controlled trials that screening is not associated with adverse psychological consequences at the population level [7]. Furthermore, screen-detected patients have high levels of modifiable risk factors and therefore have the potential to benefit from early intervention [8]. There is some evidence of benefit associated with general health or cardiovascular disease (CVD) risk screening in population-based samples. Participants randomised to general practitioner (GP)- or nurse-led interventions focussing on lifestyle factors have demonstrated modest reductions in CVD risk scores and risk factors over $1[9,10]$ and 5 years, respectively [11]. To date, however, there is little evidence of the impact on mortality of a population-based screening programme for diabetes. Effects on population mortality provide a strong basis for evaluation of early detection by screening and subsequent treatment. Modelling studies have suggested that significant reductions in diabetes-related mortality of $26-40 \%$ could be achieved by a 4 -yearly [12] or 5-yearly diabetes screening regimen [13]. However, there is no evidence from prospective studies to support these findings.

We assessed mortality over 18 years in a population-based cohort of 4,936 individuals who were divided into three groups. In 1990-1992, one-third of the cohort was randomly selected for invitation to 5 -yearly screening for type 2 diabetes by OGTT and related CVD risk factors. In 2000 2003, a further third of the cohort was randomly selected for invitation to diabetes screening. The remaining third were not invited for screening during any stage of the follow-up period. In two separate analyses we compare the mortality of (1) individuals who were invited to the 1990-1992 screening round and those who were not, over 10 years of follow-up (1990-1999); and (2) individuals who were invited to the 2000-2003 screening round and those who were not, over 8 years of follow-up (2000-2008). We also examined the mortality of attenders vs non-attenders among those who were invited in each screening round.

\section{Methods}

The Ely study (Cambridgeshire, UK) was established in 1990 as a prospective study of the aetiology of type 2 diabetes. Full details of the population are reported elsewhere [14]. In brief, approximately one-third $(n=$ $1,705)$ of all men and women aged 40-65 years old was randomly selected from a sampling frame of adults free of known diabetes registered with a single practice serving Ely $(n=4,936)$. Housebound individuals were excluded prior to invitation. Selected individuals were invited between 1990 and 1992 for screening for type 2 diabetes with a $75 \mathrm{~g}$ OGTT and related CVD risk factors. Further follow-up of this group occurred at a median of 4.5 years (1994-1996) and 10 years (2000-2003), including invitation to nonattenders at baseline (Fig. 1). At each screening round, GPs were informed by letter of participants' fasting plasma cholesterol and triacylglycerol values, blood pressure and the results of the OGTT. Among the remaining two-thirds of the sampling frame who were still alive in 2000-2003, 1,577 individuals were randomly selected for invitation to diabetes screening. The remaining 1,425 people were not invited for diabetes screening at any stage in the follow-up period. No standard intervention package was specified for people found to have type 2 diabetes or elevated CVD risk factors following screening. GPs were informed of the results and advised to take whatever action they thought necessary.

In order to assess the impact of invitation to screening on population mortality, all individuals in the original sampling frame, including those who were not invited for screening, were flagged for death certification at the Office of National Statistics (ONS). Vital status has been obtained for the entire cohort and we report results for follow-up to 31 January 2008. Deaths were coded into three groups (cardiovascular, cancer and other) based on the primary cause of death using the International Classification of Diseases, $10^{\text {th }}$ edition (ICD-10; www.who.int/classifications/icd/en/). Two researchers independently coded the deaths with $94 \%$ agreement. Consensus was reached after discussion with a third researcher. Cardiovascular death was defined as an ICD-10 code in the range I00-I99 and cancers deaths as C00-D48. It was also noted whether diabetes was included as the underlying cause of death on each certificate.

Data were available on age at baseline, sex and postal address code. Missing postcodes from the original 1990 address data were updated by a Royal Mail recommended company (www.dataprocessing.co.uk, accessed 22 June 2010). Postcodes were available for $90 \%$ of participants and were linked to enumeration districts to calculate the Townsend Index - a composite measure of material deprivation based on four factors derived from the 1991 UK census (unemployment, overcrowding, car ownership and home 
Fig. 1 The Ely study population

${ }^{\text {a }}$ Note that values do not add up to 3,231 as 229 participants in these groups died before the 2000-2003 screening round

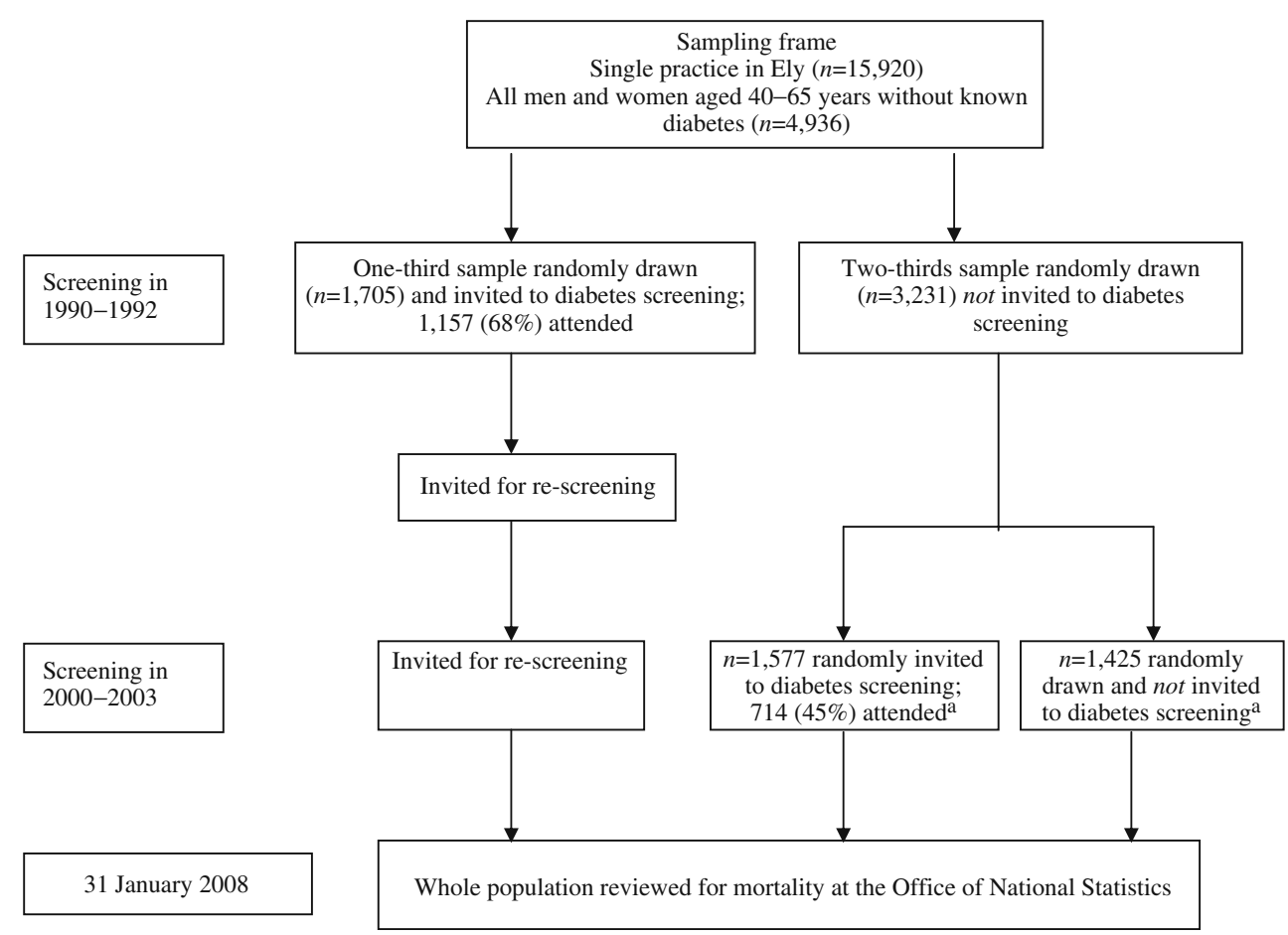

ownership). The index is a standardised $z$-score, which represents local deprivation relative to mean deprivation in England and Wales; a score above 0 implies that deprivation is greater than the mean for England and Wales, while a score below 0 indicates less deprivation [15].

Statistical analysis Baseline characteristics were summarised separately in invited and non-invited groups using means and percentages. We compared groups using the unpaired $t$ test for continuous variables and $\chi^{2}$ test for categorical data. Cox proportional HRs were calculated for the association between baseline characteristics and mortality. Kaplan-Meier survival curves were generated for the first 1990-1999 follow-up period: (1) individuals who were invited but did not attend the 1990-1992 screening round, (2) individuals who were invited and attended the 19901992 screening round; and (3) individuals who were not invited in the 1990-1992 screening round. After exclusion of deceased individuals, we replicated these analyses by comparing individuals invited and not invited for screening in 2000-2003 in the 2000-2008 follow-up period. Sensitivity analyses were undertaken by imputing the lowest and then the highest recorded values of the Townsend Index in those participants with missing data for this variable. We also excluded those participants whose death certificates reported diabetes to examine if this changed the statistical significance or direction of any of the associations.

All analyses were completed using Stata Version 10.1 (STATA Corp., College Station, TX, USA). The Ely study was approved by the Cambridge Local Research Ethics Committee (99/246). Participants gave informed consent prior to screening; approval under Section 60 of the UK Health and Social Care Act 2001 was obtained before receiving mortality information from the ONS.

\section{Results}

Baseline characteristics for the study population are shown in Table 1. Individuals who were invited for the 1990-1992 round of screening were significantly older, lived in more deprived areas and were more likely to be female than those in the non-invited group. Of those invited, 1,157 (68\%) attended for the initial screening test. There was no significant difference in age between those who did and

Table 1 Baseline characteristics of the entire Ely cohort by invited and non-invited screening groups in $1990(n=4,936)$

\begin{tabular}{llll}
\hline Baseline characteristic & $\begin{array}{l}\text { Not invited } \\
\text { for screening }\end{array}$ & $\begin{array}{l}\text { Invited for } \\
\text { screening at } \\
\text { Phase I }\end{array}$ & $\begin{array}{l}p \text { value for } \\
\text { difference }\end{array}$ \\
\hline $\begin{array}{l}\text { Total, \% }(n) \\
\text { Sex }\end{array}$ & $65.5(3,231)$ & $34.5(1,705)$ & - \\
Male, \% (n) & $50.7(1,639)$ & $45.1(769)$ & $<0.001$ \\
$\begin{array}{l}\text { Age at entry (years) } \\
\text { Female }\end{array}$ & $51.2(7.3)$ & $52.8(7.8)$ & $<0.001$ \\
$\begin{array}{l}\text { Male } \\
\text { Townsend Index } \\
\text { of deprivation }\end{array}$ & $-1.5(1.7)$ & $-1.3(2.0)$ & $<0.001$ \\
\hline
\end{tabular}

Values are mean (SD) unless otherwise stated 
did not attend. Fewer men took up the initial offer of screening than women $(45 \%$ and $55 \%$, respectively; $p<$ $0.001)$ and those who attended lived in less deprived areas than those who did not attend $(p=0.005)$. Attendance was lower in the group invited for screening only in 2000-2003 $(714 / 1,577,45 \%)$ and did not differ by sex $(p=0.74)$. Individuals who attended were younger than those who did not attend $(p<0.001)$, and they lived in less deprived areas $(p=0.02)$.

Diabetes diagnosis and overall mortality In the population invited to screening in 1990-1992, 51 participants were diagnosed with diabetes in the first round of screening, 26 in the second round and a further 31 in the final round. There were a total of 345 deaths in the whole cohort between 1991 and 1999, a median of 10 years $(47,854$ person-years of risk). There were 116 deaths among the 1,705 individuals in the group invited for screening; 52 $(45 \%)$ of the deaths were recorded as cancer-related, 41 (35\%) were due to cardiovascular causes and $23(20 \%)$ were coded as 'other'. Among the 3,231 individuals who were not invited to screening, there were 229 deaths; 107 (47\%) were cancer deaths, 74 (32\%) were CVD-deaths and $48(21 \%)$ were coded as 'other' causes. For 22 individuals (6\%) among the total deceased, diabetes was included as the underlying cause on the death certificate. In the population of people invited for screening in the 20002003 round and those who were not invited at any stage, there was a total of 291 deaths between 2000 and 2008, a median of 8.1 years $(23,144$ person-years of risk). There were 165 deaths among the 1,577 individuals in the group invited for screening; 44 (27\%) of the deaths were recorded as cancer-related, $68(41 \%)$ were due to cardiovascular causes and $53(32 \%)$ were coded as 'other'. Among the 1,425 individuals who were not invited to screening, there were 126 deaths; 47 (37\%) were cancer deaths, 43 (34\%) were CVD deaths and $36(29 \%)$ were coded as 'other' causes. There were 22 individuals (8\%) among the total deceased for which diabetes was included as the underlying cause on the death certificate.

Screening and mortality in 1990-1990 Table 2 shows the association between baseline characteristics and mortality for the period 1990 to 1999 . Mortality was independently associated with increasing age, male sex, and greater deprivation. Compared with those who were not invited, individuals who were invited to the 1990-1992 screening round had a non-significant $21 \%$ lower overall mortality (HR 0.79 [95\% CI 0.63, 1.00], $p=0.05$ ) after adjustment for age, sex and deprivation. Compared with participants who were not invited for screening in 1990-1992, mortality was significantly lower in those taking up the offer of screening (HR 0.54 [95\% CI 0.40-0.74]), and higher in those who were invited but did not attend (HR 1.36 [95\% CI 1.011.82]) (Fig. 2). Over the same period, the average age at death was significantly higher for men invited for screening in 1990-1992 than for those not invited (64.1 and 61.0 years, respectively, $p=0.01)$. The difference among women was smaller and did not achieve statistical significance (63.5 and 61.7 years, respectively, $p=0.17$ ).

Screening and mortality in 2000-2008 For the period 2000 to 2008 , there was no significant difference in mortality between invited and non-invited groups in the 2000-2003 screening round (HR 1.18 [95\% CI 0.93-1.51]) (Table 3). Again, mortality was significantly lower in those taking up the offer of screening in 2000-2003 (HR 0.52 [95\% CI $0.35,0.78]$ ) and higher in those who were invited but did not attend (HR 1.73 [95\% CI 1.34-2.24]) compared with participants who were not invited (Fig. 3).

Sensitivity analyses for missing data on deprivation did not influence the direction of the association between invitation and mortality. However, the strength and statistical significance of the association between invitation for screening and mortality increased after imputing the lowest recorded values of the Townsend Index for deprivation $(p=$ 0.04) in the 1990-1992 screening round. Exclusion from the analysis of participants whose death certificates reported diabetes did not alter the statistical significance or direction of any of the associations.

\section{Discussion}

Invitation to type 2 diabetes and related CVD risk factor screening in 1990-1992 was associated with a non-
Table 2 Cox proportional HRs and $95 \% \mathrm{CI}$ for the association between baseline characteristics and mortality; Ely cohort, UK, 1990-1999 $(n=4,936)$

\footnotetext{
${ }^{a}$ Adjusted for age, sex and deprivation

${ }^{\mathrm{b}}$ Compared with those not invited in 1990-1992
}

\begin{tabular}{lll}
\hline Baseline characteristic & Crude HR $(95 \% \mathrm{CI})$ & Adjusted $^{\mathrm{a}} \mathrm{HR}(95 \% \mathrm{CI})$ \\
\hline Age (years) & $1.10(1.08-1.12)$ & - \\
Male sex & $1.58(1.27-1.96)$ & - \\
Townsend Index of deprivation & $1.08(1.02-1.14)$ & - \\
Invitation to screening in 1990-1992 $^{\mathrm{b}}$ & $0.96(0.77-1.20)$ & $0.79(0.63-1.00)$ \\
Invited/attenders $^{\mathrm{b}}$ & $0.64(0.47-0.86)$ & $0.54(0.40-0.74)$ \\
Invited/non-attenders $^{\mathrm{b}}$ & $1.68(1.27-2.22)$ & $1.36(1.01-1.82)$ \\
\hline
\end{tabular}




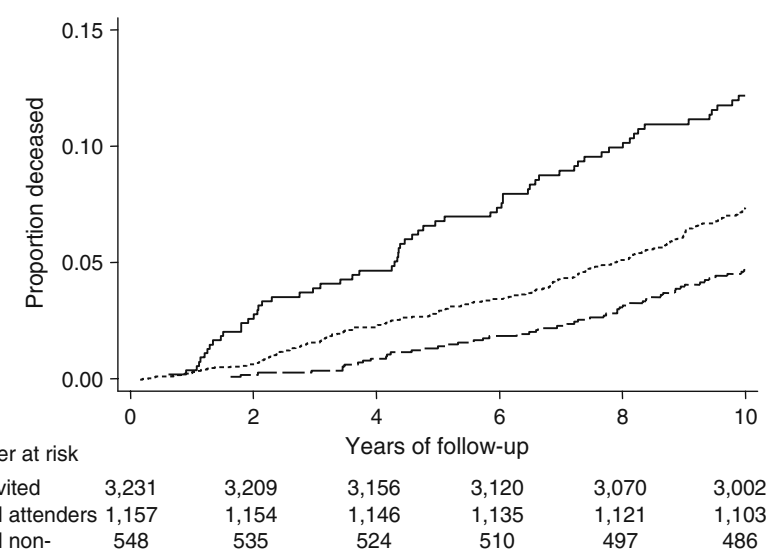

Fig. 2 Kaplan-Meier survival curves for the Ely cohort, UK, 19901999, by three groups: individuals who were invited but did not attend screening in 1990-1992 (solid line), individuals who were invited and attended screening in 1990-1992 (long-dashed line); and individuals who were not invited for screening in 1990-1992 (short-dashed line). There were 345 deaths over a median of 10 years

significant $21 \%$ reduction in all-cause mortality in the middle-aged population of Ely between 1990 and 1999. In the second stage of follow-up (2000-2008), there was no significant difference in the mortality risk between participants who were invited for screening in 2000-2003 and those who were not invited. Compared with the non-invited group, participants who attended for screening at any time point had a significantly lower mortality and those who did not attend had a significantly higher mortality.

The point estimate for the reduction in all-cause mortality between 1990 and 1999 attributable to the invitation for screening is slightly smaller than the benefits suggested by modelling studies $[12,13]$. This is unsurprising as the variables used in these simulation models are different from those in the Ely study, e.g. screening test, frequency of testing, length of follow-up, outcome (diabetes-related mortality) and choice of population subgroups in which screening should be undertaken. Conversely, the estimated reduction in all-cause mortality in the invited group is higher than might be expected from the findings of other studies that have examined general health or cardiovascular risk screening in population-based samples [9-11]. In these studies, participants were randomised to GP- or nurse-led interventions focussing on lifestyle factors and demonstrated modest reductions in CVD risk scores and risk factors over $1[9,10]$ and 5 years, respectively [11]. The reduction in mortality in Ely was seen even without specification of a standard intervention package for people with newly diagnosed type 2 diabetes or elevated CVD risk factors. The studies are also different as participants in the Ely study have been followed up for a much longer period of time (10 and 8 years) and we have data on hard clinical endpoints rather than modelled risk of events. The $21 \%$ reduction in mortality in the invited group is higher than that seen for most public health interventions and is comparable to the reduction in mortality achieved by population screening programmes for breast cancer [16] and abdominal aortic aneurysm [17]. As mortality was even lower in participants who attended their screening appointment, this suggests that invitation to screening for type 2 diabetes and associated CVD risk factors between 1990 and 1999 may have had a substantial public health effect, although we cannot rule out the possibility that this was a chance finding.

We have no information about underlying mechanism in this pragmatic study. It is unlikely that the difference between invited groups was due to the early identification of diabetes in Ely participants alone. Very few of the deaths were ascribed to diabetes as an underlying cause and only a relatively small proportion of the population was diagnosed with diabetes. The screening process may therefore have impacted in other ways on mortality in the invited group. As screened individuals had information on CVD risk factors reported to their general practitioner, this may have resulted in greater attention being paid by patients and practitioners to risk factor management including prescription of drug treatment for primary prevention and lifestyle change. Such health promotion activities and lifestyle changes could impact on multiple health outcomes and the benefits would not be restricted simply to those individuals found to have type 2 diabetes. Alternatively, the difference between groups at baseline may have introduced selection bias. Despite the random selection of participants into invitation groups, participants who were offered screening were older at baseline, lived in more deprived areas and included a smaller proportion of men.
Table 3 Cox proportional HRs and $95 \%$ CI for the association between baseline characteristics and mortality; Ely cohort, UK, 2000-2008 $(n=3,002)$

\footnotetext{
${ }^{a}$ Adjusted for age, sex and deprivation

${ }^{\mathrm{b}}$ Compared with those not invited in 2000-2003
}

\begin{tabular}{lcl}
\hline Baseline characteristic & Crude HR (95\% CI) & Adjusted $^{\mathrm{a}}$ HR (95\% CI) \\
\hline Age (years) & $1.12(1.10-1.14)$ & - \\
Male sex & $1.58(1.25-2.00)$ & - \\
Townsend Index of deprivation & $1.09(1.02-1.16)$ & - \\
Invitation to screening in 2000-2003 $^{\mathrm{b}}$ & $1.20(0.95-1.51)$ & $1.18(0.93-1.51)$ \\
Invited/attenders $^{\mathrm{b}}$ & $0.46(0.31-0.69)$ & $0.52(0.35-0.78)$ \\
Invited/non-attenders $^{\mathrm{b}}$ & $1.85(1.45-2.36)$ & $1.73(1.34-2.24)$ \\
\hline
\end{tabular}




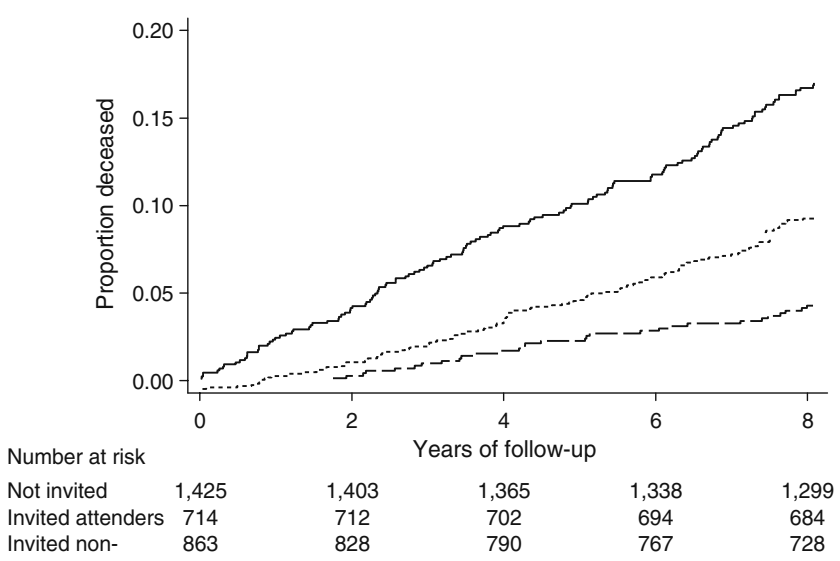

attenders

Fig. 3 Kaplan-Meier survival curves for the Ely cohort, UK, 20002008, by three groups: individuals who were invited but did not attend screening in 2000-2003 (solid line), individuals who were invited and attended screening in 2000-2003 (long dash); and individuals who were not invited for screening in 2000-2003 (short dash). There were 291 deaths over a median of 8 years

The older age and greater level of deprivation might be associated with a higher mortality, while the higher proportion of women could be associated with a lower mortality in this group. As a result, there is no consistent direction for possible selection bias. Furthermore, although the differences were statistically significant, they were not large and the results are presented following adjustment for these baseline differences. Such adjustment does not rule out residual confounding nor does it account for factors on which we have no measurement. For example, it was not possible to adjust for BMI, smoking, serum lipids, blood pressure, or the treatment of CVD risk factors, since information was not available on the entire cohort of people, only those who attended for screening. In contrast to many studies of screening, it is unlikely that there was any significant effect from lead or length time bias as mortality was assessed for the whole population from baseline.

The finding for the association of invitation to screening and mortality for those people invited to screening in 2000 2003 differs from that seen in the people invited in 19901992. Fewer people attended for screening in 2000-2003; this may have contributed to the lack of association with mortality in the second phase of the study. It is likely that opportunistic case finding (CVD risk assessment and diabetes testing) was more widely practiced during 2000-2008 than in the earlier phase of the study (1990-1999). Furthermore, statin use for primary prevention may have become more widespread, perhaps attenuating differences between the invited and non-invited groups. A survivor cohort effect may also have played a role. Preventable deaths may have occurred between 1990 and 1999 in the unscreened group, so the second phase of screening may have missed those individuals for whom invitation to screening may have resulted in reduced risk. One of the challenges of assessing mortality in the long term is that health and social contexts change, which limits interpretation of findings. Finally, the moderate sample size means that we may have been underpowered to detect possible differences in mortality between screened and unscreened groups.

The proportion of deaths due to cancer, CVD and other causes was similar in the invited and non-invited groups in the period 1991-1999. Between 2000 and 2008, the proportion of deaths due to CVD appears to be higher in the invited compared with the non-invited group. This difference could be due to chance, and/or changes in health behaviours and prescribing patterns that are associated with mortality from other causes such as cancer.

Participants who attended for screening had a significantly lower mortality than those who did not attend in both phases of screening. This is likely to be due, at least in part, to the 'healthy volunteer effect', which has been observed in a number of epidemiological studies [18-20]. Analysis of attendance in a long-term prospective cohort, the British Regional Heart study, showed that non-attenders were older than attenders, had higher blood pressure, were more likely to smoke, were more likely to work in manual occupations, and had significantly increased mortality rates [21]. Similarly, non-attenders in the Family Heart study exhibited an adverse risk profile [22]. In the Ely cohort, individuals who attended for screening were from less deprived areas than those who did not attend. Our study also supports previous literature showing that men are less likely to attend for diabetes screening than women [23], which has implications for the organisation and delivery of CVD and type 2 diabetes screening programmes. Our findings suggest that special efforts may need to be made to target deprived men, or screening programmes could contribute to a worsening in the social gradient of health.

As with all screening programmes, benefit is influenced by uptake. In this study, the highest mortality rates were found in non-attenders. Careful attention to identification of these individuals and to strategies to maximise uptake may contribute to a greater reduction in mortality. In order to reduce the level of non-attendance, more pragmatic means of identifying individuals at high risk of type 2 diabetes rather than the OGTT are needed. Indeed, mass screening for diabetes using the OGTT alone is impractical because of the associated logistical challenges and the poor reproducibility of the test [6]. $\mathrm{HbA}_{1 \mathrm{c}}$ might be considered as a suitable screening tool in this context as it is a relatively inexpensive measure, has good reproducibility, it does not require fasting, and hence can be taken at any time of the day.

Finally these data also demonstrate that observational studies are rarely truly 'observational' and that participation in research with the associated measurement and feedback of results may have important potential effects. 
Strengths and limitations The Ely cohort is a large, population-based cohort study with robust outcome measures and long-term follow-up. The use of death certificate information to identify cause of mortality may be affected by inaccuracies in classification. However, errors in classification would not be systematically different between the two groups and would not affect all-cause mortality. Furthermore, it is unlikely that death registration would be incomplete in this cohort as indicated by the very low loss to tracking by the ONS $(0.25 \%)$. All-cause mortality is a robust measure of the magnitude of impact that a screening programme might have in a particular population. It is an all-inclusive measure that addresses both direct and indirect (for example false reassurance) effects of a screening programme. It puts disease-specific mortality reduction in the context of other competing risks and gives the screened individual an estimation of the overall benefit to be reasonably expected [24]. The study population was almost entirely white, which limits the generalisability of our findings. We are unable to assess if participants in the group who were not invited for screening at baseline were subsequently screened for type 2 diabetes on an opportunistic basis (following recommendations from organisations such as Diabetes UK [25]). This may have led to an attenuation of the observed association.

Conclusion These data suggest that invitation to screening for type 2 diabetes and related CVD risk factors may have been associated with a reduction in mortality in the Ely cohort between 1990 and 1999, albeit not quite reaching statistical significance. Between 2000 and 2008 there was no significant reduction in mortality between invited and non-invited groups. Taken together, these results suggest that, while there may be some overall benefit associated with screening, this may have been greater when fewer primary prevention activities were being undertaken in primary care and also when screening people aged 40 to 65 rather than 50 to 75 years. These results should be interpreted with some caution because of the differences at baseline between groups, the lack of information on potential residual confounders, the lack of information about mechanism, and the apparent contrasting effects of the offer of screening in the two time periods. Nevertheless, a strategy of population screening for diabetes and CVD risk factors, as recently proposed for the NHS [5] could potentially have an important impact.

While results from this analysis are promising, it remains unclear whether early detection and treatment of diabetes and related CVD risk factors is beneficial and produces sufficient improvement in long-term health outcomes to justify the economic costs. In view of the extensive organisational, technical and financial input that a national type 2 diabetes screening programme would demand, evidence of the cost-effectiveness of screening from prospective randomised controlled trials [26] prior to implementation would be desirable.

Acknowledgements The Ely study was supported by the Medical Research Council and NHS Research and Development (R\&D). M. Rahman was funded by an NHS R\&D training fellowship.

Duality of interest The authors declare that there is no duality of interest associated with this manuscript.

\section{References}

1. International Diabetes Federation (2009) The Diabetes Atlas, 4th edn. IDF, Brussels

2. King H, Aubert RE, Herman WH (1998) Global burden of diabetes, 1995-2025: prevalence, numerical estimates, and projections. Diabetes Care 21:1414-1431

3. Harris MI, Klein R, Welborn TA, Knuiman MW (1992) Onset of NIDDM occurs at least 4-7 yr before clinical diagnosis. Diabetes Care 15:815-819

4. UKPDS (1990) UK Prospective Diabetes Study 6. Complications in newly diagnosed type 2 diabetic patients and their association with different clinical and biochemical risk factors. Diabetes Res 13:1-11

5. Department of Health (2008) Putting prevention first. Vascular checks: risk assessment and management. DOH, London

6. Waugh N, Scotland G, McNamee P, et al. (2007) Screening for type 2 diabetes: literature review and economic modelling. Health Technol Assess 11: iii-iv, ix-xi, 1-125

7. Eborall HC, Griffin SJ, Prevost AT et al (2007) Psychological impact of screening for type 2 diabetes: controlled trial and comparative study embedded in the ADDITION (Cambridge) randomised controlled trial. BMJ 335:486

8. Echouffo-Tcheugui JB, Sargeant LA, Prevost AT et al (2008) How much might cardiovascular risk be reduced by intensive therapy in people with screen-detected diabetes? Diabet Med 25:1433-1439

9. Family Heart Study Group (1994) Randomised controlled trial evaluating cardiovascular screening and intervention in general practice: principal results of British family heart study. Family Heart Study Group. BMJ 308:313-320

10. OXCHECK (1995) Effectiveness of health checks conducted by nurses in primary care: final results of the OXCHECK study. Imperial Cancer Research Fund OXCHECK Study Group. BMJ 310:1099-1104

11. Engberg M, Christensen B, Karlsmose B, Lous J, Lauritzen T (2002) General health screenings to improve cardiovascular risk profiles: a randomized controlled trial in general practice with 5-year follow-up. J Fam Pract 51:546-552

12. Kuo HS, Chang HJ, Chou P, Teng L, Chen TH (1999) A Markov chain model to assess the efficacy of screening for non-insulin dependent diabetes mellitus (NIDDM). Int J Epidemiol 28:233-240

13. Chang HJ, Kuo HS, Tung TH, Chou P, Chen TH (2000) Evaluation of a population-based screening for type 2 diabetes: a communitybased screening project in Puli, Taiwan. Prev Med 31:396-402

14. Williams DR, Wareham NJ, Brown DC et al (1995) Undiagnosed glucose intolerance in the community: the Isle of Ely Diabetes Project. Diabet Med 12:30-35

15. Townsend P, Phillimore P, Beattie A (2004) Health and deprivation: inequality and the north. Croom Helm, London

16. Blanks RG, Moss SM, McGahan CE, Quinn MJ, Babb PJ (2000) Effect of NHS breast screening programme on mortality from 
breast cancer in England and Wales, 1990-8: comparison of observed with predicted mortality. BMJ 321:665-669

17. Ashton HA, Buxton MJ, Day NE et al (2002) The Multicentre Aneurysm Screening Study (MASS) into the effect of abdominal aortic aneurysm screening on mortality in men: a randomised controlled trial. Lancet 360:1531-1539

18. Benfante R, Reed D, MacLean C, Kagan A (1989) Response bias in the Honolulu Heart Program. Am J Epidemiol 130:1088-1100

19. Lindsted KD, Fraser GE, Steinkohl M, Beeson WL (1996) Healthy volunteer effect in a cohort study: temporal resolution in the Adventist Health Study. J Clin Epidemiol 49:783-790

20. Froom P, Melamed S, Kristal-Boneh E, Benbassat J, Ribak J (1999) Healthy volunteer effect in industrial workers. J Clin Epidemiol 52:731-735

21. Thomas MC, Walker M, Lennon LT et al (2002) Non-attendance at re-examination 20 years after screening in the British Regional Heart Study. J Public Health Med 24:285-291
22. Davies G, Pyke S, Kinmonth AL (1994) Effect of non-attenders on the potential of a primary care programme to reduce cardiovascular risk in the population. Family Heart Study Group. BMJ 309:1553-1556

23. Sargeant LA, Simmons RK, Barling RS et al (2010) Who attends a UK diabetes screening programme? Findings from the ADDITION-Cambridge study. Diabet Med 27:995-1003

24. Black WC, Welch HG (1997) Screening for disease. Am J Roentgenol 168:3-11

25. Diabetes UK (2006) Early identification of people with type 2 diabetes. Position statement. Available at: www.diabetes.org.uk, accessed 15 August 2010

26. Echouffo-Tcheugui JB, Simmons RK, Williams KM et al (2009) The ADDITION-Cambridge trial protocol: a cluster randomised controlled trial of screening for type 2 diabetes and intensive treatment for screen-detected patients. BMC Public Health 9:136 